\title{
Fine Tuned Seed Mediated Synthesis and Photothermal Response of Gold Nanorods.
}

\author{
Byron D. Gates $^{1 *}$, Iris Guo ${ }^{1}$, Taeeun Chung ${ }^{1}$ \\ 1. Department of Chemistry, Simon Fraser University, Burnaby, BC, Canada; (*email: bgates@sfu.ca)
}

Gold nanomaterials are of widespread interest for the potential to assist in applications that include the delivery and selective release of drugs, hyperthermia based treatment of cancers, and enhanced image contrast in biological systems. ${ }^{1}$ These nanomaterials can be prepared in a variety of shapes, sizes, and surface chemistries as appropriate for these and other applications. One reason to tune these parameters is to adjust their plasmon resonant properties [1]. For example, tuning the longitudinal band of their plasmon resonance, one can tune the overlap between this resonance and an incident laser source. Both can be tuned to fit in the so-called "water window" of absorbance in biological tissues; this region of low absorbance by tissues and water spans much of the near-infrared wavelengths of the electromagnetic spectrum.

Methods to create gold nanomaterials with the desired near-infrared plasmonic properties include coreshell preparations, gold nanocages, and nanorods. The gold nanorods are of particular interest for the studies presented herein. The selection of gold nanorods includes the ability to tune their plasmonic properties through controlling the aspect ratio (length to width ratio) during their synthesis. Recent developments in the synthesis of seed mediated synthesis of gold nanorods include an increased yield of nanorods, relative to other by products, such as spherical nanoparticles and other shapes [2-4]. These studies have demonstrated that the molecular composition and, thus, properties of the additive(s) in solution during the growth of the nanorods has a significant influence on their final shape and purity. Furthermore, the time of addition of certain additives can also further influence these properties of the syntheses.

Gold nanorods prepared by a seed mediated synthesis in the presence of a precisely controlled mixture of surfactants proved to be a suitable method for tuning the location of the longitudinal plasmon resonance of these materials. The concentrations of some additives have, however, not been optimized although their collective properties have a high yield on the synthesis of gold nanorods. A number of the components of this synthesis have been fine-tuned through further studies and will be discussed in this presentation. One example is the demonstration that the addition of a trace additive at part-per-thousand levels that influences the ability to fine tune the location of near-infrared plasmon resonant band of these gold nanorods (Figure 1). Another aspect that will be covered in this presentation is the influence of further additives that tune the kinetics and electrochemical potential of the solution during the initial stages of nanorod growth. These results have further implications in tuning the purity and aspect ratio of these syntheses. The products of these syntheses are following through a series of analytical methods, such as extinction spectroscopy, transmission electron microscopy or TEM (including a detailed statistical analysis), high resolution TEM, and elemental analyses (e.g., energy dispersive X-ray spectroscopy).

The photothermal response of the gold nanorods prepared by through a modified seed mediated method is explored in this presentation for their potential utility in hyperthermia and triggered release of small molecules. The photothermal response is triggered through excitation with a near-infrared laser tuned to 
overlap with the longitudinal plasmon resonant band of the gold nanorods. The products on this photothermal induced process were monitored by STEM and HRTEM analyses, and the changes to the products distinguished through a quantitative statistical analysis. This detailed analysis was used to create a model for the shape and size evolution of these nanomaterials as a function of energy delivered from the laser. The results of this analysis yield a new proposed mechanism for photothermal response of these nanorods, which will be quantitatively compared to the results of systems prepared without the use of the additives. These results have implications in how these materials could be utilized in photothermal processes, such as for triggering molecular release and hyperthermia treatments.

A systematic investigation of the parameters that influence the growth of gold nanorods through a seed mediated synthesis with the addition of trace additives will be presented, along with the photothermal response of gold nanorods as a function of the types of additives used in preparing these materials [5].

\section{References:}

[1] M.-C. Daniel, D. Astruc, Chem. Rev., 104(1) (2004), p. 293.

[2] X. Ye, et al, ACS Nano, 6 (2012), p. 2804.

[3] X. Ye, et al, Nano Lett., 13 (2013), p. 765.

[4] X. Ye et al, Nano Lett., 13 (2013), p. 2163.

[5] We acknowledge funding support from the Natural Sciences and Engineering Research Council (NSERC) of Canada, the Canada Research Chairs Program (B.D. Gates), and CMC Microsystems through the MNT Financial Assistance program that facilitated access to materials characterization services. This work made use of 4D LABS shared facilities supported by the Canada Foundation for Innovation (CFI), British Columbia Knowledge Development Fund (BCKDF), Western Economic Diversification Canada, and Simon Fraser University.
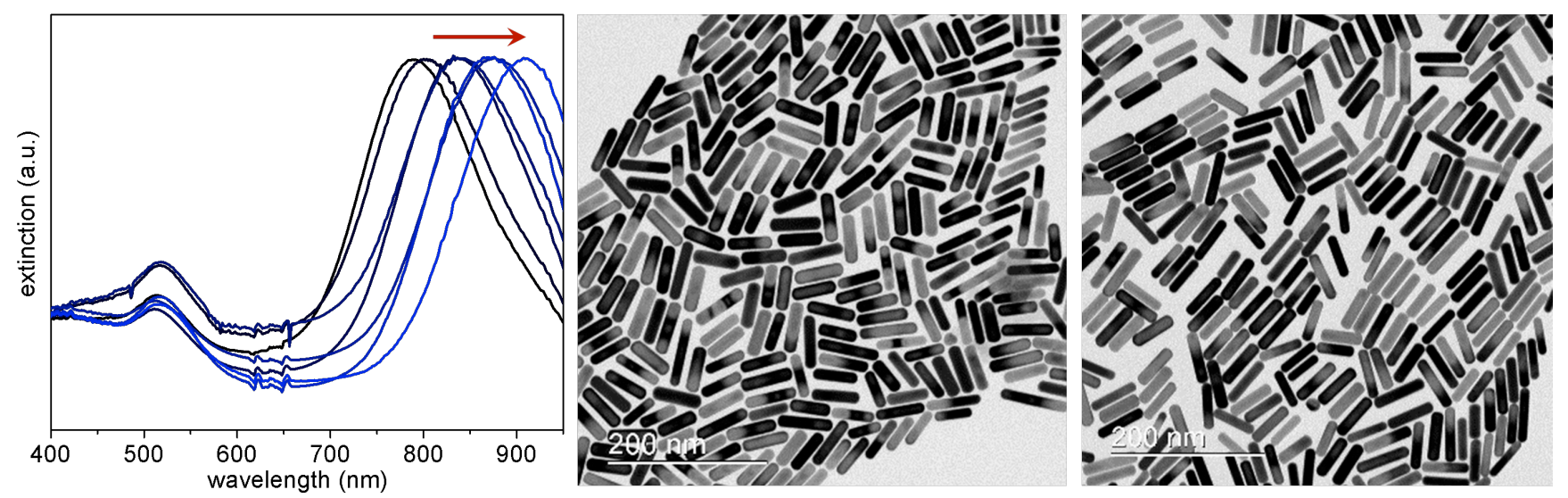

Figure 1. Extinction spectroscopy of gold nanorods with a progressive increase in aspect ratio corresponding to a step-wise red shift of the longitudinal plasmon band. Transmission electron microscopy analyses were used to assess changes in the aspect ratio, dimensions, and purity of the products of this fine-tuned synthesis. 